\title{
An unusual case of upper gastro intestinal bleeding in a adolescent female
}

Keywords: mucocutaneous pigmentation, duodenal bulb, mucosa, gastrointestinal tract, gi endoscopy

\section{Short communication}

A 12year old female patient presented in paediatric emmergency with history of upper gastrointestinal bleeding for 2days with severe anaemia and associated mucocutaneous pigmentation. Two units of blood transfusion was given to the patient. Upper GI Endoscopy revealed well defined polyps in pyloric antrum, larger one prolapsing into pyloric antrum from duodenal bulb suggestive of Peutz Jeghers Hamartomatous polyp (Figure 1). On single contrast barium meal examination a small well defined lobuted filling defect is found in pyloric antrum of stomach. Another large filling defect is seen in duodenal bulb causing expansion of it and protruding into antropyloric region (Figure 2).

Peutz-Jeghers syndrome is a rare, AD disease linked to a mutation of the STK 11 gene which is characterized by the development of benign hamartomatous polyps in the gastrointestinal tract in association with a hyperpigmentation on the lips and oral mucosa. These polyps are also at risk of acute gastrointestinal bleeding, intussusception and bowel obstruction. ${ }^{1}$ In PJS polyps can be located anywhere in the gastrointestinal tract, most commonly seen in the small bowel (70-90\%), the colon $(50 \%)$ and the stomach $(25 \%){ }^{2}$ Solitary Peutz-Jeghers polyps are generally have their origin in the small bowel, duodenum, colon, rectum and are extremely rare in the stomach, with only 8 well documented cases being reported to date. ${ }^{3} \mathrm{PJS}$ also known as periorificial lentiginosis with an incidence of $1 / 200,000$ live-borns. The most frequent complication at young age is recurrent intussusception due to multiple hamartomatous polyps, usually in the small intestine. ${ }^{4}$

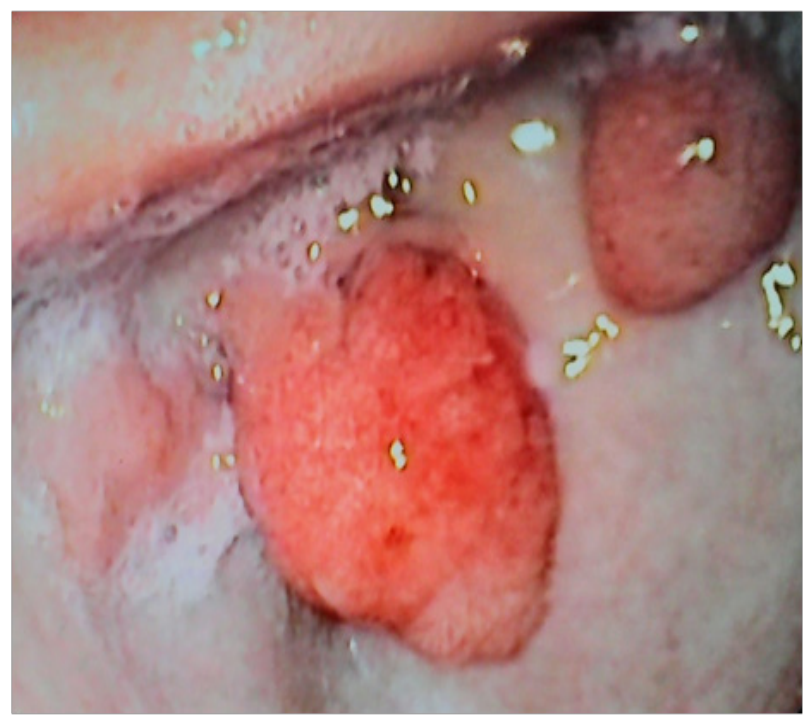

Figure I On upper Gl Endoscopy well defined polyps are seen in pyloric antrum, larger one prolapsing into pyloric antrum from duodenal bulb.
Volume 2 Issue 5 - 2017

\author{
Debi U,' Lal S,' Srikanth KP,' Prasad KK, ${ }^{3}$ \\ Thapa $\mathrm{BR}^{2}$ \\ 'Division of GE Radiology, Department of Superspeciality of \\ Gastroenterology, India \\ ${ }^{2}$ Division of Pediatric GE, Department of Superspeciality of \\ Gastroenterology, India \\ ${ }^{3}$ Division of GE Histopathology, Department of Superspeciality \\ of Gastroenterology, India
}

\section{Correspondence: Uma Debi, Division of GE Radiology,} Department of Superspeciality of Gastroenterology, Postgraduate Institute of Medical Education and Research, Chandigarh (UT) -160 012, India, Tel +9 I I722756603, $+919417526614, \mathrm{Fax}+911722744401,2744450$, Email debi_uma@yahoo.co.in

Received: March 31, 2017 | Published: April II, 2017

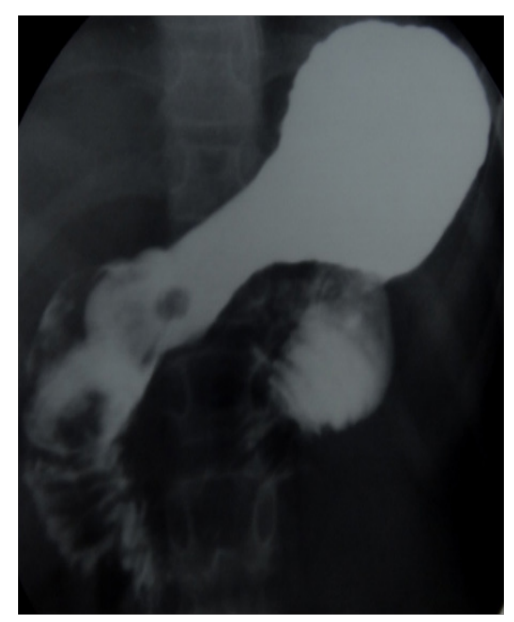

Figure 2 On single contrast barium meal examination a large filling defect is seen in duodenal bulb which is protruding into antropyloric region. Another small well defined lobuted filling defect is also found in pyloric antrum of stomach.

\section{Acknowledgements}

None.

\section{Conflict of interest}

Author declares that there is no conflict of interest.

\section{References}

1. Tomas C, Soyer P, Dohan A, et al. Update on imaging of Peutz-Jeghers syndrome. World J Gastroenterol. 2014;20(31):10864-10875. 
2. Turpin A, Cattan S, Leclerc J, et al. Hereditary predisposition to cancers of the digestive tract, breast, gynecological and gonadal: Focus on the Peutz-Jeghers. Bull Cancer. 2014;10(9):813-822.

3. Lunca S, Porumb V, Velenciuc N, et al. Giant solitary gastric Peutz-Jeghers polyp mimicking a malignant gastric tumor: the largest described in literature. J Gastrointestin Liver Dis. 2014;3(3):321-324.
4. Chae HD, Jeon CH. Peutz-Jeghers syndrome with germline mutation of STK11. Ann Surg Treat Res. 2014;86(6):325-330. 